\title{
Epistemología de la Educación Física: principales interrogantes y desafíos ${ }^{1}$
}

An epistemology of physical education: main questions and challenges

\author{
Valter Bracht*
}

\section{Resumen}

\begin{abstract}
El artículo pretende ofrecer un pequeño panorama de las discusiones epistemológicas de nuestra disciplina en Brasil, que en parte por las características idiosincrásicas, mantiene relación o es influenciada por las discusiones que sobre el tema adelantan otros países; estas discusiones contienen la naturaleza del saber que fundamenta la práctica social de la Educación Física. Esta disciplina busca una autonomía en relación con otras áreas del saber a través de la construcción de un universo simbólico de legitimación propia.
\end{abstract}

Palabras clave: Epistemología, educación física, naturaleza del conocimiento, posibilidad de conocimiento científico.

\begin{abstract}
In this article, a general view about the epistemological discussions of our discipline in Brazil is offered. This view is partly related to or influenced by the discussions carried out on the topic in other countries, because of idiosyncratic features. These discussions hold the knowledge nature establishing the practice of Physical Education. This discipline seeks autonomy in relation with other areas of knowledge, building a symbolic universe that legitimizes it.
\end{abstract}

Key words: $\quad$ Epistemology, physical education, nature of knowledge, possibility of scientifical knowledge.

Fecha de recepción: 12 de octubre de 2007

Fecha de aceptación: 10 de noviembre de 2007

1 Traducción: Dana Milena Chávarro Bermeo. Socióloga, profesora de la Facultad de Educación Física de la Universidad Pedagógica Nacional. danachabe@yahoo.com

* Doctor de la Universidad de Oldenburg, Alemania. Profesor de la Universidad Federal do Espiritu Santo. Autor de Educación Física y ciencia: Cenas de un casamiento (in) feliz. 


\section{Introducción}

Al constituirse como campo social en el siglo XIX y más fuertemente en el inicio del siglo XX (particularmente a partir de los sistemas educacionales), la educación física establece un conjunto de prácticas y acumula y organiza un conjunto de conocimientos (incluyendo ahí el saber hacer), configurando de ésta forma un cuerpo teórico. Con la institucionalización y crecimiento de este campo hay un proceso creciente de diferenciación interna, pues los agentes sociales del campo pasan a desempeñar papeles también diferenciados. Un momento importante en este proceso es la identificación y aceptación de la necesidad de formar un profesional específico para actuar en este campo ${ }^{2}$ : la formación específica del profesor de educación física. Con eso, surgieron instituciones formadoras de profesores para desempeñarse en diferentes campos sociales, principalmente en los sistemas educacionales y deportivos.

La constitución, también como campo académico, deriva de las necesidades de su propia intervención social, pero también de su inserción en la enseñanza superior (universitaria o no), contribuye para la institución en el campo de prácticas típicas de la cotidianidad académica: la realización de congresos, publicación de revistas científicas (en sustitución de las revistas técnicas), la realización de cursos de posgrados, incluso maestrías y doctorados. Aunque ese desarrollo es a nivel mundial, se viene generando con tiempos y especificidades locales.

La discusión epistemológica en la disciplina está focalizada en la discusión de varios aspectos: 1 . De la naturaleza del conocimiento que fundamenta esta práctica de la educación física; 2 . De la posibilidad de que el conocimiento científico oriente con seguridad esa intervención, y 3. De la necesidad o la posibilidad de que la educación física se constituya como ciencia autónoma o, también de la identidad epistemológica de esta área.

La educación física moderna nace en un caldo cultural, en un "paisaje cognitivo" (Nachmanovich, 2001) demasiado optimista en cuanto al potencial de la racionalidad científica. En el ambiente del siglo XIX había poco espacio para las preguntas sobre la necesidad y la posibilidad de una educación física científica y racional. No obstante, el término "educación física científica y racional" o "gimnasia científica y racional", estaba presente en muchos títulos de libros de finales del siglo XIX e

\footnotetext{
2 Anteriormente, los instructores de gimnasia eran oriundos y formados/ entrenados en otros campos, principalmente el militar o, en su defecto, los profesores recibían un pequeño entrenamiento para realizar los cursos de educación física.
}

inicios del XX. De éste modo, es interesante recordar que los primeros métodos de educación física se basaban fundamentalmente en un conocimiento anatómico y fisiológico del cuerpo y del movimiento corporal, que (pre)concebían el cuerpo como una entidad del mundo de la naturaleza, cuyo funcionamiento obedecía a leyes naturales (no en vano se pretendía "amoldar" a través de lo físico el carácter de los jóvenes). El conocimiento científico tiene el potencial de orientar una intervención también racional y científica en el sentido de optimizar su funcionamiento.

\section{El paradigma hegemónico de la producción del conocimiento en la educación física y su cuestionamiento}

La producción del conocimiento o el conocimiento que fundamenta la educación física (particularmente aquella que asume el credo cientificista) fue esencialmente el producido por disciplinas de las ciencias naturales, ya que el cuerpo y la actividad física realizada por aquel fueron considerados, en la esfera del cartesianismo, elementos de la pura naturaleza (en contraposición a la cultura o al espíritu). Ese conocimiento que pretendía sustentar la intervención social desde la educación física, dado que no está constituido en el campo propio de la educación física, proviene de las disciplinas científicas emergentes, particularmente la anatomía (kinesiología) y la fisiología (en algunos casos la biometría). Esa perspectiva es también victoriosa en el campo de la medicina, y como la educación física fue abrazada por ella (colaborando con su legitimidad social), esto cambió, también, el conocimiento legítimo en el campo emergente de la educación física.

Cuando el campo de la educación física se desarrolló al punto de bosquejar iniciativas para asumir una función de producir conocimiento para orientar (académicamente) su intervención social, los intelectuales de la educación física asumen mayoritariamente la perspectiva hegemónica que es la de las ciencias naturales. En el caso brasileño, particularmente en la década de 1970, cuando el campo de la educación física comienza a hacer parte del sistema de posgraduación y de ciencia y tecnología como un área autónoma, la investigación y la producción académica del área estaban fuertemente orientadas por la óptica disciplinar, por lo que se convino en llamar ciencias madres (Fisiología del ejercicio, Psicología del Deporte, Sociología del Deporte, etc.). 
Esa producción de conocimiento provocó lo que llamé en otro momento (Bracht, 1999) despedagogización del teorizar en la educación física en favor de una cientificidad de ese teorizar, basado en el modelo de las ciencias naturales. Es el momento histórico en el que las llamadas Ciencias del Deporte se presentan internacionalmente como una opción muy fuerte para la organización del campo del conocimiento, unido tradicionalmente a la educación física. En muchos países esa denominación (y la concepción en ella presente) fue oficialmente adoptada (Sportwissenschaft en Alemania, Sports Sciences en los EE. UU. $)^{3}$; en otros casos, se habla de kinesiología o Ciencia de la Motricidad Humana.

La perspectiva dominante era la de intelectuales formados en otros campos, particularmente en disciplinas científicas como la fisiología, o en derivaciones como la Biomecánica (física), el aprendizaje motriz (psicología del aprendizaje), produjeron conocimientos que pudiesen ser utilizados para fundamentar la educación física. Entre tanto, intelectuales de otros campos no tenían más horizonte inmediato que la intervención, o bien porque no pensaban a partir del campo de la educación física, y sí pensaban la educación física a partir de sus campos disciplinares. Creció mucho la producción del conocimiento, pero muy poco el diálogo, se estableció entre los conocimientos disciplinares y también hubo poco diálogo entre ese conocimiento y aquellos que estaban en la intervención (escaso impacto obtuvo ese conocimiento en la práctica pedagógica de los profesores) ${ }^{4}$. En función de la importancia social y política del fenómeno deportivo, la educación física escolar fue instrumentalizada para servir al sistema deportivo (pirámide deportiva), o fue puesta en un plano secundario en lo concerniente a la producción del conocimiento. O sea, la producción del conocimiento con reconocimiento social capaz de proveer capital simbólico a los investigadores era aquella que interesaba al desarrollo del sistema deportivo.

Ese modelo fue muy criticado, algunos entendieron que la educación física necesitaba identificar específicamente un objeto de conocimiento propio para lograr autonomía en cuanto campo académico, a semejanza de otras disciplinas científicas. De ahí a la reivindicación de ella como una nueva disciplina científica fue un paso corto.

\footnotetext{
3 En Brasil, en 1979 se funda el Colegio Brasileño de Ciencias del Deporte, casi un clon del American Collage of Sports Sciences.

4 Así mismo porque esos profesionales "hablaban" lenguajes muy diferentes.
}

La reivindicación por una autonomía científica en la forma de una nueva disciplina tenía como objetivo principalmente, lograr o mantener el estatus universitario para esa área, es decir una forma de legitimarse en el mundo universitario académico, lo que sería alcanzado mas fácilmente en la medida en que se pudiese hablar de una nueva ciencia (Ciencia Deportiva, Ciencias del Deporte, Ciencia de la Motricidad Humana, Ciencias del Movimiento Humano, Kinesiología, etc.).

Por otro lado, una serie de divisiones socioculturales en los años 1970 y 1980 (que no cabe discutir en este trabajo), redundó en el "descubrimiento" de las prácticas corporales (deporte, danza, gimnasia) como objeto de estudio importante de las ciencias sociales y humanas (Sociología, Historia, Filosofía). Esa "ampliación" del conocimiento sobre las prácticas corporales, ahora bajo o a través de las ciencias humanas, provocó un interrogante en el campo de la educación física sobre la hasta entonces hegemonía de las ciencias naturales. Esto propició un mayor interrogante también de la práctica social, de la intervención social hecha por la educación física, vista de forma positiva y positivista hasta entonces.

Una de las principales consecuencias de ese movimiento fue el cuestionamiento del imperio de la "naturaleza" presente en el campo de la educación física. El discurso científico que colocaba a nuestro objeto en el mundo de la naturaleza, confiere a la educación física un carácter de intervención racional-pedagógica en el cuerpo (y en el carácter, en el espíritu) mediante ejercicios físicos. Así, la educación física se configuró a partir de un conocimiento que se colocaba como universal y abstracto, naturalizando su necesidad y existencia. Con base en esta postura epistemológica, la identidad de la educación física fue entendida como algo preexistente y también inmutable, fijo, terminado; si quisiéramos, inscrita en la naturaleza o en la necesidad que está inscrita en la naturaleza. Se trataba entonces de descubrir tal naturaleza o identidad. El instrumental teórico-metodológico de las ciencias humanas permitió reedificar esa visión de la educación física afirmando que la educación física es una construcción sociohistórica, en última instancia, política; por tanto, no hay identidad al ser descubierta, pero sí posibilidades de construcción de sentido, las cuales son condicionadas socioculturalmente. A partir de esa perspectiva, las diferentes identidades posibles se enfrentan históricamente en la disputa por la hegemonía y legitimidad en el proceso de construcción de sentido. 
En el ámbito específico de la Educación Física escolar en Brasil, ese cambio podría ser caracterizado como un giro culturalista en el sentido de que el objeto de enseñanza de la educación física pasa a ser entendido, no como algo de la naturaleza, sino, como algo del mundo de la cultura. Una perspectiva de educación física pasa a ser entonces la de que la educación física escolar es responsable de la transmisión de una parte de la cultura, afirmándose un concepto de Cultura Corporal o Cultura del Movimiento (pero siempre cultura) que abriga diferentes manifestaciones históricas como el deporte, la danza, la gimnasia, las luchas, etc.

\section{Identidad epistemológica y producción de conocimiento: principales desafíos}

Una de las principales dificultades enfrentadas por el área en relación a la construcción de una autonomía académico-científica es la posibilidad de identificar y construir un objeto propio de investigación, y también un abordaje metodológico propio. Entiendo que tales dificultades se derivan en parte de un sentido equivocado del objeto de estudio. Un ejemplo de eso es la idea de que el deporte o la actividad física podrían equivaler a un objeto de una nueva disciplina académica. Si bien el deporte puede ser objeto de estudio de una "nueva" disciplina científica, continuará siendo objeto de investigación de la sociología o de la fisiología. Esa dificultad ya se expresa en la duda de denominaciones como ciencia del deporte o ciencia del movimiento humano en singular, o en plural. Es decir, un objeto de una disciplina académica no es simplemente un recorte de lo real o un fenómeno empírico, es, por el contrario, una construcción a partir de interrogantes y de un interés guiador del conocimiento propio.

Así, entiendo que el asunto es construir una problemática o problemáticas teóricas compartidas que puedan reunir una o más comunidades académicas que comulguen con la creencia de que tal problemática es significativa y merece ser objeto de investigación y construcción de un cuerpo teórico -que vale la pena jugar el juego--. En ese sentido no descarto la posibilidad de constitución de una comunidad de investigación en torno a la problemática teórica del aumento del rendimiento deportivo, iniciativa que, tengo certeza, sería bien vista por el propio sistema deportivo.

Para la educación física, entendida como práctica pedagógica, entiendo que una problemática teórica que podría ser compartida y articular una comunidad académica, sería la de investigar la participación y con- tribución del movimiento humano y sus objetivaciones culturales en la educación del hombre.

Otro problema, que en Brasil es factor de división de la comunidad académica ligada a la Educación Física, es aquello de que muchos trabajan con la idea de entender la Educación Física primordialmente como actividad académica y otros la entienden preferentemente como actividad de intervención ${ }^{5}$. Los primeros buscan un objeto científico, los segundos buscan un objeto que caracterice la intervención social propia de la educación física. Esa segunda posición maneja una transformación de la lógica predominante: en vez de mirar el objeto a partir de la disciplina madre, se construye un objeto a partir de la Educación Física, utilizando los recursos teórico-metodológicos disponibles por las disciplinas académicas. Esa transformación propiciaría un paso importante en el sentido de articular la producción del conocimiento en el plano académico, con la intervención (con la práctica de los profesores de Educación Física en distintos sectores).

En relación con la convocatoria de este seminario: ¿es posible una epistemología para la construcción disciplinar que oriente la enseñanza, la proyección cultural y las políticas públicas de la Educación Física? Diría que no se trata de construir una epistemología. Entiendo más interesante pensar en la importancia de la actividad epistemológica para el área, como propone Fensterseifer (2006: 31). Para este autor, en lugar de buscar una epistemología para la educación física, deberíamos asumir la "actividad epistemológica" como práctica importante para el campo, no en el sentido de afirmar la ecuación de la tradición moderna de verdad=razón=ciencia, y sí de cuestionarla. La "naturaleza de la epistemología" importaría menos de lo que su ejercicio por parte de aquellos que hacen y están dispuestos a discutir su actividad y su presencia en los diferentes espacios que habitan; como también sus relaciones con la contemporaneidad.

Por otro lado, la idea de "actividad" garantiza la noción de proceso que acompaña algo que está vivo, que se repone siempre que nuevas discursividades que se instalan en el ámbito de nuestra área; tanto por aquellos que inauguran temáticas, como por aquellos que las releen bajo nuevos lentes teóricos o nuevos paradigmas. Tarea hermenéutica interminable en cuanto existen intérpretes dispuestos a interferir en el debate. (Fensterseifer, 2006: 33)

\footnotetext{
5 Parlebas también se refiere a ésta cuestión y, tanto él como yo, entendemos que la educación física debe ser pensada primordialmente como una práctica de intervención con orientación académica, aunque Parlebas halle posible construir algo como la praxiología.
} 
Reitero, que una sociedad plural, que adopta en gran medida un anti-fundamentalismo (o la reflexividad en el sentido de Beck y Giddens), no parece loable pensar en la educación física de una gran unidad en torno de un proyecto de educación física, la hegemonía es siempre objetada y resistida.

En relación a la orientación de las políticas públicas para el sector de la educación física y del deporte, hay un conflicto de intereses entre una ciencia crítica y autónoma y el sistema deportivo que opera con las reglas del mercado. Eso genera una tensión. El sistema deportivo está interesado en su reproducción y crecimiento y, por tanto, en conocimiento que lo auxilie en esa dirección. Un conocimiento que critica el sistema deportivo puede antagonizar con los intereses de la institución deportiva. Lo mismo vemos en el financiamiento de investigaciones por grandes empresas que normalmente prohíben la divulgación de conocimientos que son contrarios a sus intereses. De ahí, la importancia de un sistema público de investigación que permita que él mismo realice su actividad con autonomía financiera y política.

Uno de los problemas para la constitución de comunidades que comparten una problemática teórica dentro del "gran campo de la educación física" (incluyendo, por ejemplo, la comunidad ligada al deporte) es el reconocimiento de esa comunidad por el sistema de ciencia y tecnología de un determinado país, principalmente por parte del sistema público de financiamiento de la producción científica y de la formación de profesionales.

Hasta el momento hablamos de la actividad epistemológica en la educación física en la perspectiva de estudio (científico) del movimiento humano, o sea, de un conocimiento sobre el movimiento humano, pero ¿sería posible hablar de un conocimiento a partir del movimiento?, Lo que conocemos por movimiento? En 116 el plano mas específico de nuestro objeto, el movimiento humano, uno de los desafíos resulta del reconoci-

\section{Referencias}

Betti, M. (1994). O que a semiótica inspira ao ensino de educação física. Discorpo, 3, 25-45. São Paulo.

Bracht, V. (1999). Educação Física \& ciência: cenas de um casamento (in)feliz. Ijuí: EditoraUnijuí.

Bracht, V. y Crisório, R. (orgs.) (2003). A educação física no Brasil e na Argentina: Identidades, desafios y perspectivas. Campinas: Autores Associados. miento del cuerpo como "sujeto epistemológico" vale decir como "sujeto de conocimiento". En la perspectiva que fundó la tradición de la educación física, el cuerpo y el movimiento corporal, fueron siempre "objeto" (de estudio, de manipulación) de una razón capaz de analizarlo, diseccionarlo, dominarlo y moldearlo, operación posible por asumir el cuerpo como mera "res extensa". A partir del giro culturalista y otros desarrollos (psicoanálisis y fenomenología al frente), ese conocimiento no se sustentó, debido a que se probó que el cuerpo no es mero "objeto" del proceso histórico. Expresiones como el cuerpo habla, el cuerpo piensa, el cuerpo siente, son indicadores de ese cambio de estatus epistemológico y ontológico del cuerpo ${ }^{6}$. Así, uno de los desafíos que se pone para la "actividad epistemológica" en la Educación Física es: si el cuerpo además de objeto de manipulación de conocimiento racional científico, es también, sujeto de la acción, ¿ ¿ómo se relacionan razón y cuerpo en el movimiento? ¿cuáles "conocimientos" del mundo son posibles en el moverse? ${ }^{7}$ No tendríamos que dedicar especial atención a la educación de la sensibilidad, a una educación estética?

En Brasil, varios caminos han sido transitados buscando avanzar en esa dirección: a partir de la hermenéutica filosófica de Gadamer (Fensterseifer, Cuerpo y Lenguaje); la semiótica particularmente la de C. Peirce (Betti, 1994) y la teoría estética.

Lo práctico es intelectual, la cultura física es intelectual y la cultura intelectual es física. Se debe reconocer que El cuerpo humano es más que un instrumento, materia, o máquina.

El encargo social de la educación física es precisamente el bienestar de la sociedad.

Finalmente, sólo con estudio e investigación rigurosa la educación física y la cultura física pueden ser reconocidas en el ámbito de la comunidad académica y científica. (DD
Fensterseifer, P. (2006). Atividade epistemológica e educação física. In: Nóbrega, T.P. (org.). Epistemologia, saberes e práticas da educação física. João Pessoa: Editorial Universitária, p. 29-37.

Nachmanovitch, D (2001). O sujeito encarnado: questões para pesquina no/do cotidiano. Rio de Janeiro: DP\&A.

\footnotetext{
6 Ver al respecto la ya citada obra de Nachmanovich (2001).

En otro momento (Bracht, 1999), discutiendo esta cuestión formulé el desafío de la siguiente forma: "Ni movimiento sin pensamiento, ni movimiento y pensamiento y si, movimiento-pensamiento".
} 